\title{
A comparative analysis of survival rate and shear bond strength of bondable molar tubes with two different mesh designs
}

\author{
Punia $K^{1}$, Shetty $D^{2 *}$, Sharma $P^{3}$, Kumar $P^{4}$, Raza $M^{5}$, Jain $S^{6}$ \\ ${ }^{1}$ Former PG Student, ${ }^{2,4}$ Professor, ${ }^{3}$ Professor and Head, ${ }^{5,6}$ Senior Lecturer, Dept. of Orthodontics and Dentofacial Orthopedics, ITS-Centre \\ for Dental Studies and Research, Muradnagar, Ghaziabad, Uttar Pradesh, India \\ *Corresponding Author: Shetty D \\ Email: divyashetty@its.edu.in
}

\begin{abstract}
Objective: To evaluate and compare the shear bond strength (in vitro) and survival rate (in vivo) of molar tubes with conventional and modified mesh base designs.

Materials and Methods: Molar tubes of two different base design ie conventional (American Orthodontics) and modified base design (Ortho Organizers) were evaluated and compared for shear bond strength and survival. 20 molar tubes of each type were bonded on freshly extracted first permanent molars. Shear bond strength was tested using a Universal testing machine. 20 patients requiring fixed orthodont ic treatment were selected. Molar tubes with two different base designs were placed on alternate sides in the four quadrants so that each arch had molar tubes of both types. Survival of the bonded tubes was evaluated monthly for a period of six months.

Results: Molar tubes having conventional mesh base design showed greater shear bond strength (10.49 Mpa) as compared to modified mesh base design $(10.41 \mathrm{Mpa})$. The difference was found to be statistically significant $(\mathrm{p}=0.004)$. However, tubes with a modified mesh base (OO) showed a greater survival rate (95\%) compared to those with conventional mesh base (85\%).

Conclusion: Molar tubes with conventional mesh base design bonded with Transbond XT showed greater shear bond strength than molar tubes with modified mesh base design. Molar tubes with modified mesh base design showed a greater survival rate than molar tubes with conventional mesh base design.
\end{abstract}

Keywords: Shear bond strength, Molar tubes, Universal testing machine.

\section{Introduction}

In recent years, the orthodontic specialty has almost totally switched to the bonding appliance considering the advantages like aesthetics, hygiene, comfort and ease of application it provides over the banded appliances. ${ }^{1-2}$

Bonding of attachments to molars rather than banding is a less frequently adopted practice. Molar tubes bonded with either a chemically cured or light-cured resin adhesive have exhibited failure rates over $21 \%$. Also, brackets bonded to molars have a lower bond strength and a high clinical failure rate than brackets bonded to teeth located more anteriorly in the arch. ${ }^{3} \mathrm{High}$ bond failure rates reported may be from orthodontic and masticatory forces or from moisture contamination during the bonding process due to isolation difficulties. Manufacturers have sought to address bond failure on molars through improvements in adhesive technology and attachment designs. Improvements include increased base dimensions and changes in the base design. ${ }^{4}$

So the present study evaluated bond strength and survival rate of bondable molar tubes with two different mesh designs. The null hypothesis stated that there was no difference in the survival rate and shear bond strength of bondable molar tubes with two different mesh designs.

\section{Materials and Methods}

This study was carried out in the Department of Orthodontics and Dentofacial Orthopaedics and Centre for Advanced Research, I.T.S Centre for Dental Studies and Research, Muradnagar.

The study was divided into two parts- survival rate (assessed in vivo) and shear bond strength (assessed in vitro). For evaluation of shear bond strength, molar tubes from two different manufacturers were obtained: Group 1IFIT TUBES (American Orthodontics) with conventional mesh base design and Group 2- Maestro series (Ortho Organiser) with modified mesh base design. To elaborate on the differences in the mesh base design, a microscopic analysis of the mesh bases was carried out using a Stereomicroscope Olympus (SZX7) and Magnus Pro Software at $40 \mathrm{X}$ magnification. The mesh space, also known as aperture area and the thickness of the mesh wire were calculated for each tube type. The mesh wire thickness was measured in three different locations and an average reading was taken in microns. The aperture area was calculated by taking the average length and breadth of the aperture base, each measured at three locations. Two specimens of each type of tube were measured and the average was taken.

40 freshly extracted permanent first molar teeth were mounted in self-cure acrylic. The blocks were divided into 2 groups with 20 samples in each group for bonding molar tubes of two different mesh base designs. The teeth were cleaned and rinsed with pumice slurry to ensure the removal of plaque and the organic pellicle. Moisture control was maintained after the rinse to provide a dry working field. The teeth were etched for 30 seconds. After the etching period the etchant was rinsed off the teeth. The teeth were dried thoroughly with a moisture and oil free air spray. A thin layer of bonding agent was painted over the etched enamel surface. The coating was thinned by a gentle air burst for 1 to 2 seconds. Molar tube placement was started immediately after all etched surfaces were coated. A thin layer of adhesive was applied on the base of the tube and 
placed on the tooth with proper alignment and position with the help of a bracket placement gauge. The excess of adhesive material was removed from the surface of the tooth using an explorer tip. Curing was done using LED curing light with a wavelength of 340-400 nm for 10 seconds at each corner of the molar tube.

\section{Bond strength test}

After bonding, all the specimens were stored in distilled water at $37^{\circ} \mathrm{C}$ for 24 hours before testing for bond strength. The shear bond strength was measured using Universal testing machine. A mounted jig i.e a steel rod with a flattened end was attached to the crosshead of universal testing machine and an occluso-gingival load was applied to the molar tube parallel to the buccal surface of the teeth.
The force required to shear off the molar tube was recorded in Newtons at a crosshead speed of $0.5 \mathrm{~mm} / \mathrm{minute}$.

\section{Adhesive remnant index}

The mode of bond failure was assessed according to the amount of adhesive left on the molar tube surface utilizing the adhesive remnant index (ARI) under stereomicrocsope (OLYMPUS, Japan, Model no -SZ2-ILST) at 10X zoom. The ARI ranges from:

0 - no adhesive remained on the tooth

1 - less than half of the enamel bonding site covered with adhesive

2 - more than half of enamel bonding site covered wih adhesive

3 - the enamel bonding site covered entirely with adhesive
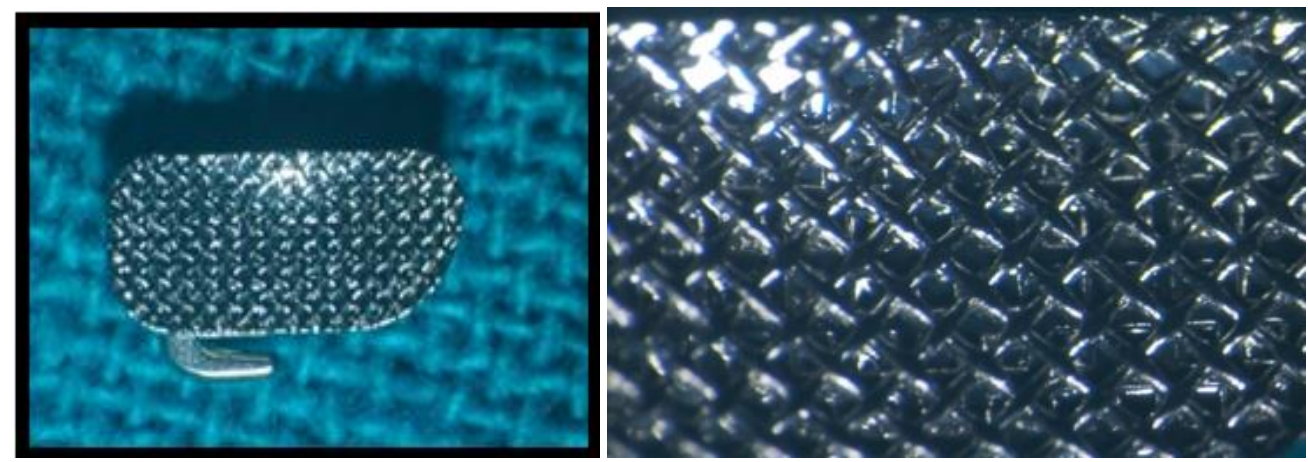

Fig. 1: Molar tube with conventional mesh base (American Orthodontics) at $10 \mathrm{x}$ and $40 \mathrm{x}$ magnification
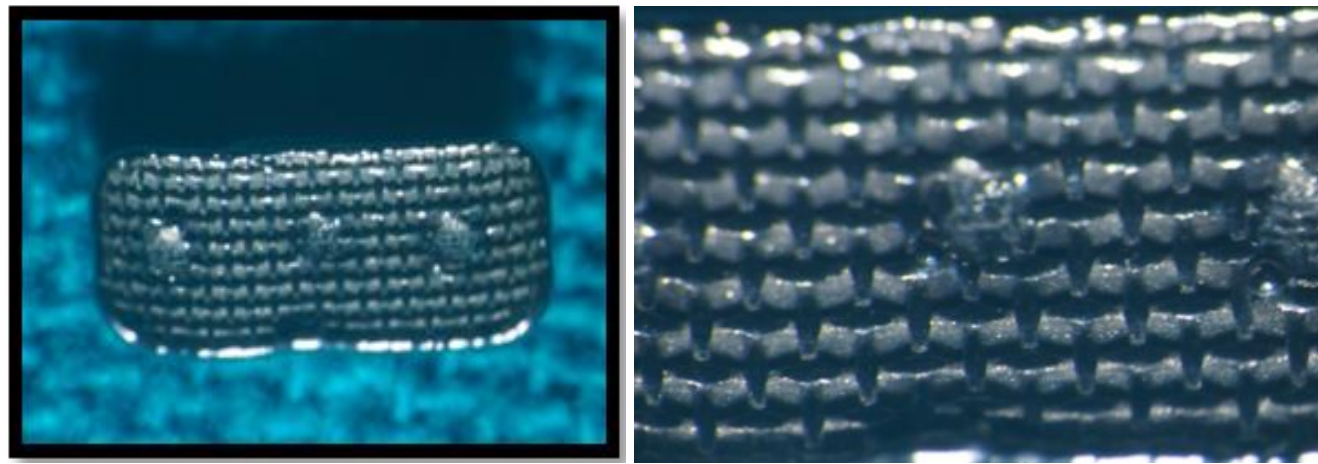

Fig. 2: Molar tube with Modified mesh base (Ortho Organizer) at 10x and $40 \mathrm{x}$ magnification
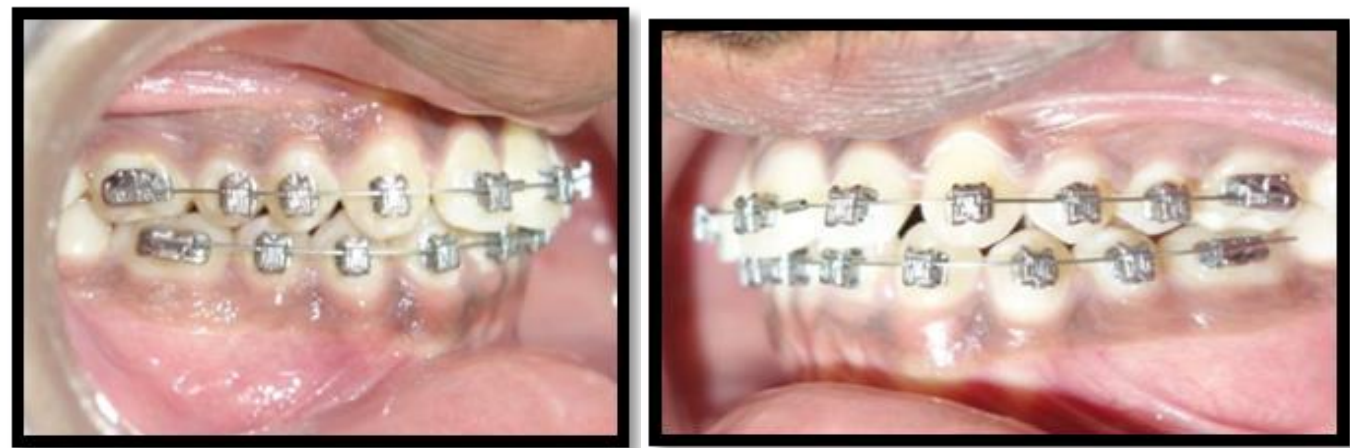

Fig. 3: (a) Showing the placement of molar tube on $1^{\text {st }}$ molar of upper right quadrant $(\mathrm{AO})$ and lower right quadrant $(\mathrm{OO})$ and (b): Showing the placement of molar tube on $1^{\text {st }}$ molar of upper left quadrant $(\mathrm{OO})$ lower left quadrant $(\mathrm{AO})$ 


\section{For the survival analysis}

20 patients (age $>12$ years) requiring fixed orthodontic treatment and with no previous history of orthodontic treatment were included in the study. Patients with cleft lip and palate, craniofacial syndromes, absence of or planned extraction of first permanent molar, $1^{\text {st }}$ permanent molar with evidence of demineralization or hypoplastic enamel or congenital defects, patients requiring orthognathic surgery, extra-oral or intra-oral anchorage reinforcement were excluded. Molar tubes with two different base designs were placed on alternate sides in the four quadrants so that each arch had molar tubes of both the types. The patients were evaluated every 4 weeks in order to assess loss of molar tubes for a total period of 6 months. All bond failures were noted on each patient's record taking into account the side (right and left), arch (maxilla and mandible), date (day/month/year) and position (tooth). The loose molar tube was replaced by new molar tubes and rebonded using direct bonding technique.

\section{Statistical analysis}

The data was subjected to statistical analysis using SPSS (Statistical Package for Social Sciences) version 20.0 statistical analysis software. The means and standard deviations of shear bond strength of the molar tubes were calculated. The student $t$ test for two independent groups was used to test difference in bond strength. The chi square test was used to compare bond failure rate in vivo. The level of significance and confidence intervals were 5\% and $95 \%$ respectively.

\section{Results}

In the present study, the shear bond strength ranged from 9.5- 10.9 Mpa in tubes of Group 1 and 9.8-11 MPa in Group 2 (Table 1). Reynolds suggested that the bond strength of 5.9 -7.8 Mpa is clinically acceptable. The bond strength of tubes in both the groups was higher than this range. However, molar tubes in Group 1 had a greater bond strength (mean $10.49 \mathrm{Mpa}$ ) compared with Group 2 (mean $10.41 \mathrm{Mpa}$ ) and the difference was statistically significant $(\mathrm{p}=0.04)$ with a mean difference in shear bond strength of $0.37 \mathrm{MPa}$.

Table 2 shows the number of breakages of molar tubes recorded every 30 days for a period of 6 months. There was a failure of a total of 8 tubes in Group 1and 5 tubes in Group 2. Group 2 showed a greater success rate of $95 \%$ as compared to Group 1. Group 1 showed a greater failure rate of $15 \%$ compared to Group 2 which showed a success rate of only $85 \%$. Survival of both the types of tubes was compared using Chi Square test (Table 3). Although the Group 2 molar tubes showed a higher success rate, the difference was not statistically significant ( $\mathrm{p}$ value .263).

Table 1: Shear bond strength of molar tubes in MPa

\begin{tabular}{|c|c|c|c|c|}
\hline Group & $\mathbf{N}$ & Mean & Std. Deviation & Std. Error Mean \\
\hline 1 & 20 & 10.490 & .3837 & .0858 \\
\hline 2 & 20 & 10.118 & .3787 & .0847 \\
\hline
\end{tabular}

\begin{tabular}{|c|c|c|c|c|c|c|c|c|c|}
\hline & \multicolumn{2}{|c|}{$\begin{array}{c}\text { Levene's Test for } \\
\text { Equality of Variances }\end{array}$} & \multicolumn{7}{|c|}{ t-test for Equality of Means } \\
\hline & \multirow[t]{2}{*}{$\mathrm{F}$} & \multirow[t]{2}{*}{ Sig. } & \multirow[t]{2}{*}{$\mathbf{T}$} & \multirow[t]{2}{*}{ df } & \multirow[t]{2}{*}{$P$ value } & \multirow[t]{2}{*}{$\begin{array}{c}\text { Mean } \\
\text { Difference }\end{array}$} & \multirow[t]{2}{*}{$\begin{array}{l}\text { Std. Error } \\
\text { Difference }\end{array}$} & \multicolumn{2}{|c|}{$\begin{array}{l}\text { 95\% Confidence } \\
\text { Interval of the } \\
\text { Difference }\end{array}$} \\
\hline & & & & & & & & Lower & Upper \\
\hline SBS & .068 & .796 & 3.086 & 38 & .004 & .3720 & .1206 & .1279 & .6161 \\
\hline
\end{tabular}

Table 2: No. of breakages over a period of 6 months

\begin{tabular}{|c|c|c|c|c|c|c|c|}
\hline Group & T1 & T2 & T3 & T4 & T5 & T6 & Total \\
\hline 1 & $1(\mathrm{Q} 1)$ & $2(\mathrm{Q} 2, \mathrm{Q} 3)$ & $1(\mathrm{Q} 2)$ & $4(\mathrm{Q} 1, \mathrm{Q} 1, \mathrm{Q} 4, \mathrm{Q} 4)$ & 0 & 0 & 8 \\
\hline 2 & $1(\mathrm{Q} 2)$ & $1(\mathrm{Q} 3)$ & $3(\mathrm{Q} 1, \mathrm{Q} 2, \mathrm{Q} 1)$ & 0 & 0 & 0 & 5 \\
\hline
\end{tabular}

Table 3: Six months survival analysis

\begin{tabular}{|c|c|c|c|c|c|}
\hline \multicolumn{6}{|c|}{ Group * Result Cross tabulation } \\
\hline & & & \multicolumn{2}{|c|}{ Result } & \multirow[t]{2}{*}{ Total } \\
\hline & & & Failure & Success & \\
\hline \multirow[t]{4}{*}{ Group } & \multirow[t]{2}{*}{1} & Count & 6 & 34 & 40 \\
\hline & & $\%$ within group & $15.0 \%$ & $85.0 \%$ & $100.0 \%$ \\
\hline & \multirow[t]{2}{*}{2} & Count & 2 & 38 & 40 \\
\hline & & $\%$ within group & $5.0 \%$ & $95.0 \%$ & $100.0 \%$ \\
\hline \multirow{2}{*}{\multicolumn{2}{|c|}{ Total }} & Count & 8 & 72 & 80 \\
\hline & & $\%$ within group & $10.0 \%$ & $90.0 \%$ & $100.0 \%$ \\
\hline
\end{tabular}




\begin{tabular}{|c|c|c|c|c|c|}
\hline \multicolumn{6}{|c|}{ Chi-Square Tests } \\
\hline & Value & df & $\begin{array}{c}\text { Asymp. Sig. (2- } \\
\text { sided) }\end{array}$ & P value & $\begin{array}{l}\text { Exact Sig. (1- } \\
\text { sided) }\end{array}$ \\
\hline Pearson Chi-Square & $2.222^{\mathrm{a}}$ & 1 & .136 & & \\
\hline Continuity Correction $^{b}$ & 1.250 & 1 & .264 & & \\
\hline Likelihood Ratio & 2.315 & 1 & .128 & & \\
\hline Fisher's Exact Test & & & & .263 & .132 \\
\hline $\begin{array}{l}\text { Linear-by-Linear } \\
\text { Association }\end{array}$ & 2.194 & 1 & .139 & & \\
\hline McNemar Test ${ }^{\mathrm{b}}$ & & & &.$^{\mathrm{c}}$ & \\
\hline $\mathrm{N}$ of Valid Cases & 80 & & & & \\
\hline \multicolumn{6}{|c|}{ a. 2 cells $(50.0 \%)$ have expected count less than 5 . The minimum expected count is 4.00 . } \\
\hline b. Computed only for a & & & & & \\
\hline
\end{tabular}

\section{Discussion}

High bond failure rates of molar tubes have been reported; these may result from orthodontic and masticatory forces or from moisture contamination during the bonding process due to isolation difficulties. ${ }^{4}$ It has been shown that molar bonding is associated with survival times almost half that of cemented bands. Forces up to 360 Newtons have been registered in molar region in young adolescents. ${ }^{5}$ The variables associated with the shear bond strength are the size, design and the surface treatment of the adhesive contact surface of the bracket base. ${ }^{5}$ To improve bond strength the bracket adhesive pads has been a focus of development. Improved adhesive systems and the refinement of bracket base design have enabled manufactures to decrease the size of bracket base without sacrificing bond strength. ${ }^{6}$ There are various bracket base designs all in attempt to optimize the mechanical bond between the bracket and the adhesive. The design of the bracket base adhesive pad has been found to be a significant factor in shear bond strength. ${ }^{5}$ Therefore, this study was conducted with the purpose of comparing survival rate (in vivo) and shear bond strength (in vitro) of $1^{\text {st }}$ molar tubes with two different mesh designs: Group 1- IFIT TUBES (American Orthodontics) with conventional mesh base design and Group 2-Maestro series (Ortho Organiser) with modified mesh base design.

The results indicated that the bond strength did not depend on the base area of the bonding pad. The tubes in Group 1 had a surface area of $9.81 \mathrm{~mm}^{2}$ which was smaller than the surface area of $13.24 \mathrm{~mm}^{2}$ of tubes in Group 2. This is in agreement with the result of Talpur et $\mathrm{al}^{4}$ who also did not find a statistically significant relationship between bond strength and base surface area. Several other studies by Banks, ${ }^{7} \mathrm{Cucu},{ }^{8}$ Purmal ${ }^{9}$ have also suggested that there is no relationship between bond success and base surface area.

Hudson et $\mathrm{al}^{5}$ suggested that variables other than size could affect the bond strength such as: the contact surface design, any treatment applied to the base of the bracket or the adhesive system. Therefore the wire diameter and the size of the aperture between mesh strands are variable and may affect shear bond strength. In our study, the aperture area (length $\mathrm{x}$ breath) $\mu \mathrm{m}^{2}$ of Group 1 tubes (American Orthodontics) was $5060 \mathrm{~mm}^{2}$ and that of tubes in Group 2 (Ortho Organizers) was $2370 \mathrm{~mm}^{2}$. The average thickness of mesh strand in Group 1 tubes was $373.60 \mu \mathrm{m}$ and that in Group 2 was $333.5 \mu \mathrm{m}$. So the Group 1 tubes had a larger aperture area but a smaller thickness of mesh strand compared with Group 2. These two factors may have contributed to the higher shear bond strength of tubes in Group 1. Similar to this result, Hudson et $\mathrm{al}^{5}$ found in their study that the tubes with the lowest bond strength (Ormco) had a base average aperture area less than half the size of average aperture areas of the tubes with the highest bond strength $(3 \mathrm{M})$, but the largest mesh strand diameter $(126.5 \mu \mathrm{m}){ }^{5}$

The other factors that may explain the difference in bond strength of the two types of tubes are the surface treatment, mesh gauge and design. Both types of tubes had a similar single 80 mesh gauge, but the Group 1 tubes had a photo-etched mesh base with the mesh criss-crossing diagonally from corner to corner and a glossy finish. In contrast, the Group 2 tubes had a mesh made separately of stainless steel with micro etched bondable pad to maximize bond adhesion and a compound contoured base design. The surface of the mesh had a step ladder appearance with a mat finish.

In the present study, the adhesive used for bonding was Transbond XT for both the groups because previous studies have shown this adhesive to generate the highest bond strength which is in agreement with the study done by Chapman. ${ }^{10}$

Because of the intrinsic limitations of in vitro studies and the inability to fully reproduce clinical conditions, prospective clinical trials are required to determine whether certain bonding materials or conditions provide more clinically acceptable than others. ${ }^{1}$ Hence the second part of this study was designed as a clinical trial to test whether the difference of bond strength found in-vitro translated to a clinical advantage. The survival rate of the two types of molar tubes was evaluated over a six-month period using a split mouth design.

In our study the overall failure rate of bondable molar tubes ranged from 15\% in Group 1 to 5\% in Group 2. Group 
2 showed a greater success rate of $95 \%$ as compared to Group 1. Although the Group 2 molar tubes showed a higher success rate of survival, the difference was not statistically significant ( $p$ value .263). Several other studies have evaluated survival of bondable molar tubes in-vivo, but the difference in variables tested make a direct comparison difficult

Geiger et al ${ }^{11}$ reported a failure rate of $12.4 \%$ to $21.1 \%$ for bonded maxillary first molar tubes. Another study that used a light-curing adhesive also reported a high failure rate $22 \%$ of upper and $20 \%$ of lower first molar tubes - even though rapid curing reduces moisture contamination. However, considering that the failure rate of first molar bands varied from $0.56 \%$ to $34.6 \%$, bonding on molars can be substituted for bands without increasing the risk of failure. ${ }^{1}$ So to conclude, the survival of bondable molar tubes depends not only on the shear bond strength but other factors such as occlusal forces, diet, patient co-operation, bonding conditions such as materials used for bonding, moisture control, etching time, access for bonding, operator's efficiency and surface morphology of the molars may also play a role. The limitations of this study include the short follow-up period which did not cover the entire treatment time. We also did not investigate failure rates according to type of malocclusion in this study. Although some researchers found significant differences in bracket failure rates among different malocclusion types another study reported no significant differences. In addition, the biting forces may vary according to gender which was not evaluated in our study.

\section{Conclusion}

The following conclusions were drawn from the study:

1. The molar tubes with a conventional base design exhibited a significantly greater shear bond strength compared with those having a modified base design.

2. The molar tubes with a modified mesh base showed a greater success rate $(95 \%)$ compared with tubes having a conventional mesh base (85\%); however the difference was not statistically significant.

3. Adhesive Remnant index analysis indicated that the majority of bond failures occurred within the adhesive.

\section{Source of funding}

None.

\section{Conflict of interest}

None.

\section{References}

1. Jung MH. Survival Analysis of Brackets and Tubes, a twelve month assessment. Angle Orthod 2014;84:1034-40.

2. Newman. Epoxy Adhesives for Orthodontic Attachment, Progress report. Am J Orthod Dentofacial Orthop $1965 ; 51: 901-12$

3. Millet DT. Bonded Molar Tubes. Angle Orthod 2001;71:380-5.

4. Talpur M, Cunningham SJ, Moles DR, Jones SP. The

Relationship between Dimensions, Force to Failure and Shear
Bond Strengths of Bondable Molar Tubes. Angle Orthod 2012;82:536-40.

5. Hudson AP, Grobler SR, Harris AMP. Orthodontic molar brackets. Int J Biomed Sci 2011;7(1):27-34.

6. Hobson RS, Mc.Cabe JF. Relationship between enamel etch characteristics and resin enamel bond strength. Br J Dent 2002;198:463-8.

7. Banks P, Macfarlane TV. Bonded versus Banded First Molar Attachments a Controlled Clinical Trial. J Orthod 2007;34:128-36.

8. Cucu M, Driessen CH, Ferreira PD. The influence of orthodontic bracket base diameter and mesh size on bond strength. S Afr Dent J 2002;57:16-20.

9. Purmal K, Chin S, Pinto J, Yin WF, Chan KG. Microbial contamination of orthodontic Buccal Tubes from Manufactures. Int J Mol Sci 2010;11:3349-56.

10. Chapman JL, Coreil MN, Armbruster PC, Du JX. Shear bond strength of molar tubes bonded with different adhesives. Aust Orthod J 2009;25(1):1-7.

11. Geiger AM, Gorelick J, Gwinnett AJ. Bond failure rates of facial and lingual attachments. J Clin Orthod 1983;17:165-9.

How to cite the article: Punia K, Shetty D, Sharma $P$, Kumar P, Raza M, Jain S. A comparative analysis of survival rate and shearbond strength of bondable molar tubes with two different mesh designs. $J$ Dent Specialities 2019;7(2):84-8. 